\title{
Geometric proofs of the two-dimensional Borsuk-Ulam theorem
}

\author{
Karl Friedrich Siburg \\ Preprint 2008-11 \\ Mai 2008
}

Fakultät für Mathematik

Technische Universität Dortmund

Vogelpothsweg 87

44227 Dortmund

$\underline{\text { tu-dortmund.de/MathPreprints }}$ 



\title{
Geometric proofs of the two-dimensional Borsuk-Ulam theorem
}

\author{
Karl Friedrich Siburg \\ Fakultät für Mathematik \\ Technische Universität Dortmund \\ Vogelpothsweg 87 \\ 44227 Dortmund
}

May 20, 2008

\begin{abstract}
We give two proofs of the two-dimensional Borsuk-Ulam theorem. One is completely elementary and does not use homology theory or the mapping degree, while the second one makes use of the recent theory of symplectic quasi-states.
\end{abstract}

\section{Introduction}

The two-dimensional Borsuk-Ulam theorem states that a continuous vector field on $\mathbb{S}^{2}$ takes the same values on at least one pair of antipodal points.

Theorem 1.1 (Borsuk-Ulam for $\mathbb{S}^{2}$ ). Let $\sigma: \mathbb{S}^{2} \rightarrow \mathbb{S}^{2}$ be the antipodal map $\sigma(x)=-x$. Then, for every continuous mapping $V: \mathbb{S}^{2} \rightarrow \mathbb{R}^{2}$, there exists at least one point $x \in \mathbb{S}^{2}$ such that $V(x)=V(\sigma(x))$.

There are several proofs of this result (as well as higher-dimensional generalizations), most of them using some kind of degree argument [Dod, DG, GP] or combinatorial methods [Fan, Had, Tuc].

The aim of this note is to present two proofs in two dimensions which rely on geometric rather than topological arguments. The fundamental idea is the same for both of them: the pair of antipodal points is characterized as the intersection of the zero sets of two real-valued functions on $\mathbb{S}^{2}$. This intersection result on $\mathbb{S}^{2}$ can then be established by either elementary arguments (see Section 3) or the recent theory of symplectic quasi-states (see Section 4).

For the historical background and a thorough mathematical discussion of the Borsuk-Ulam theorem in arbitrary dimensions, we refer to [DG, Sect. 5].

Acknowledgement: For helpful discussions on this note, I thank Hansjörg Geiges and Leonid Polterovich. 


\section{Reduction to an intersection result}

We represent the given vector field $V$ as $V=\left(V_{1}, V_{2}\right)$ with two continuous functions $V_{i}: \mathbb{S}^{2} \rightarrow \mathbb{R}$. Then the condition $V(x)=V(\sigma(x))$ is equivalent to $f_{1}(x)=f_{2}(x)=0$ for the continuous functions $f_{i}: \mathbb{S}^{2} \rightarrow \mathbb{R}$ defined by $f_{i}(x)=V_{i}(x)-V_{i}(\sigma(x))$. In other words, we are looking for intersection points

$$
x \in f_{1}^{-1}(0) \cap f_{2}^{-1}(0)
$$

with each $f_{i}$ satisfying the relation

$$
f \circ \sigma=-f
$$

because $\sigma$ is an involution, i.e., $\sigma \circ \sigma=\mathrm{id}$.

Theorem 2.1 (Intersection result). Let $f_{1}, f_{2}: \mathbb{S}^{2} \rightarrow \mathbb{R}$ be smooth functions, both satisfying (1) and having 0 as a regular value. Then $f_{1}^{-1}(0) \cap f_{2}^{-1}(0)$ contains at least one pair of antipodal points.

As a corollary, we immediately obtain Theorem 1.1 for smooth vector fields $V$. Thus, it remains to prove Theorem 1.1 in the continuous setting, taking its smooth version for granted.

For this, we approximate the continuous functions $f_{1}$ and $f_{2}$ by smooth functions $f_{1, n}$ and $f_{2, n}$, respectively, such that each $f_{i, n}$ possesses 0 as a regular value and satisfies

$$
\left\|f_{i}-f_{i, n}\right\|_{C^{0}} \leq \frac{1}{n}
$$

for every $n \in \mathbb{N}$; see [Hir]. Now define

$$
\tilde{f}_{i, n}(x):=\frac{f_{i, n}(x)-f_{i, n}(\sigma(x))}{2} .
$$

In view of (2) we conclude that

$$
\left\|f_{i}-\tilde{f}_{i, n}\right\|_{C^{0}}=\frac{1}{2}\left\|f_{i}-f_{i, n}-\left(f_{i} \circ \sigma-f_{i, n} \circ \sigma\right)\right\|_{C^{0}} \leq \frac{1}{n} .
$$

Applying Theorem 2.1 to $\tilde{f}_{i, n}$, we obtain sequences of antipodal points $x_{n}, \sigma\left(x_{n}\right)$ such that

$$
\tilde{f}_{i, n}\left(x_{n}\right)=\tilde{f}_{i, n}\left(\sigma\left(x_{n}\right)\right)=0
$$

for all $n$. By the compactness of $\mathbb{S}^{2}$ we may assume that $x_{n} \rightarrow x$ so that the continuity of $f_{i}$ implies $f_{i}(x)=f_{i}(\sigma(x))=0$ for $i=1,2$. This finishes the proof of Theorem 1.1.

It remains to prove the intersection result given in Theorem 2.1. For this, let $\omega$ be the standard area form on $\mathbb{S}^{2}$, normalized such that

$$
\int_{\mathbb{S}^{2}} \omega=1
$$


According to Jordan's Curve Theorem, every embedded circle on $\mathbb{S}^{2}$ divides $\mathbb{S}^{2}$ into two domains. We call an embedded circle an equator if these two domains have equal area $1 / 2$.

The following theorem states that the zero set of a function satisfying (1) must contain an equator. Since two equators on $\mathbb{S}^{2}$ intersect in at least two points, Theorem 2.1 is an immediate consequence of Theorem 2.2. Note, however, that the two intersection points can be antipodal points themselves, so the intersection of the two equators may consist of just one pair of antipodal points (e.g., when the two equators are great circles).

Theorem 2.2 (Existence of an equator). Let $f: \mathbb{S}^{2} \rightarrow \mathbb{R}$ be a smooth function, satisfying (1) and having 0 as a regular value. Then $f^{-1}(0)$ contains an equator.

In the remaining part of the paper, we provide two proofs of Theorem 2.2, one elementary and one based on symplectic quasi-states.

\section{An elementary proof of Theorem 2.2}

Since 0 is a regular value for $f$ and $\mathbb{S}^{2}$ is compact, the zero set $f^{-1}(0)$ is the union of finitely many, pairwise disjoint, embedded circles $C_{j} \subset \mathbb{S}^{2}$; see, e.g., [GP]. Each of the circles $C_{j}$ divides $\mathbb{S}^{2}$ into two domains $D_{j}^{ \pm}$. If some $C_{j}$ is an equator, the theorem is proven.

Therefore, we assume that none of the $C_{j}$ is an equator. Then, for every $j$, the two domains $D_{j}^{ \pm}$have different areas, and we write $D_{j}^{-}$for the smaller one. Let us denote by $C$ that circle $C_{j}$ whose domain $D_{j}^{-}$has maximal area, and call $D^{ \pm}$the corresponding domains on $\mathbb{S}^{2}$. Note that

$$
\int_{D^{-}} \omega<\frac{1}{2}<\int_{D^{+}} \omega .
$$

Because of (1), $f^{-1}(0)$ is invariant under $\sigma$, so $\sigma(C)$ is also one of the circles $C_{j}$, and there are only two cases: either $\sigma(C)=C$ or $\sigma(C) \cap C=\emptyset$.

If $\sigma(C)=C$ then, since $\sigma$ preserves the non-oriented area, (3) implies that $\sigma\left(D^{ \pm}\right)=D^{ \pm}$, so $\sigma$ preserves orientation which is a contradiction. Therefore,

$$
\sigma(C) \cap C=\emptyset .
$$

Then $C$ and $\sigma(C)$ bound some annulus-like region $A \subset \mathbb{S}^{2}$; see Figure 1. We claim that

$$
\sigma(A)=A .
$$

Consistently neglecting boundaries, we see that the domain $D^{+}$consists of two parts, the annulus $A$ and another domain $D$. Since $C=\partial D^{-}$and $\sigma(C)=\partial D$, we have either $\sigma\left(D^{-}\right)=D$ or $\sigma\left(D^{-}\right)=D^{-} \cup A$, the latter being impossible since $\sigma$ preserves the non-oriented area. Hence $\sigma(D)=\sigma\left(\sigma\left(D^{-}\right)\right)=D^{-}$which implies $\sigma(A)=A$.

Finally, we will see that

$$
\left.f\right|_{A}=0,
$$



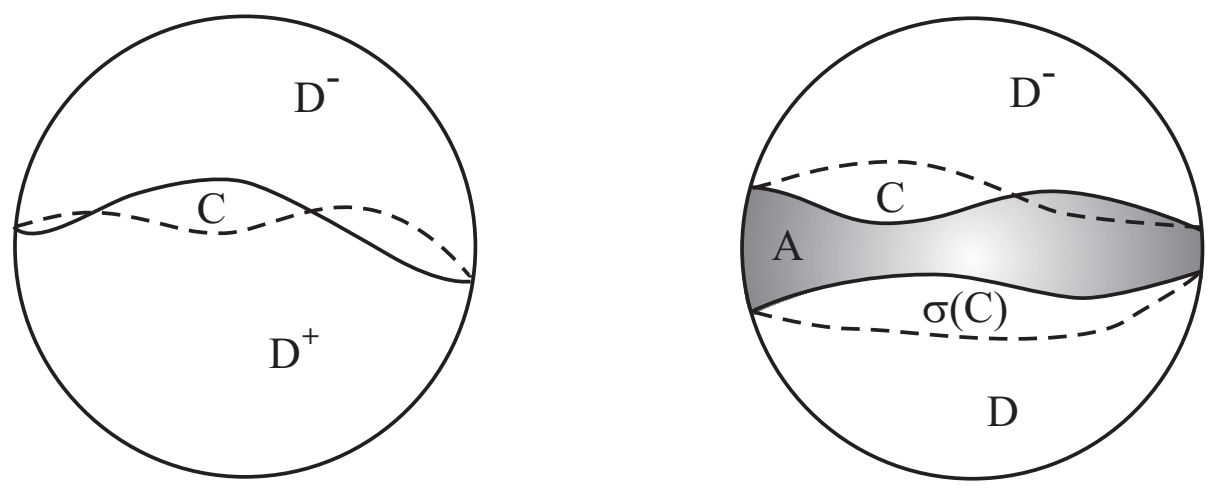

Figure 1: $\sigma(C)=C$ [left] and $\sigma(C) \cap C=\emptyset$ [right]

which, of course, is impossible because 0 is a regular value of $f$.

In order to show (4) we observe that, since $D^{-}$has maximal area, $A$ cannot contain any $C_{j}$ in its interior that is non-contractible in $A$. Now, if $A=\sigma(A)$ contains a circle $C_{j}$ which is contractible in $A$, and hence bounds an open disk $B \subset A$, then it also contains $\sigma\left(C_{j}\right)$ which bounds the open disk $\sigma(B) \subset A$. In view of (1), the function $f$ has different signs on $B$ and $\sigma(B)$. Moreover, there exists a path $\gamma:[0,1] \rightarrow A$ with $\gamma(0) \in B$ and $\gamma(1) \in \sigma(B)$ such that there are $0<a<b<1$ with $f(\gamma(t))=0$ if, and only if, $t \in\{a, b\}$. Considering the sign of $f$ along $\gamma$ one sees that this contradicts the fact that 0 is a regular value of $f$. Consequently, $A$ does not contain any $C_{j}$ in its interior, so $f$ has a fixed sign on $A=\sigma(A)$ which, in view of (1), implies (4).

Therefore, our starting assumption that none of the $C_{j}$ is an equator leads to a contradiction, proving Theorem 2.2.

\section{A symplectic proof of Theorem 2.2}

Since an area form in two dimensions is the same as a symplectic form, the above proof of Theorem 2.2 suggests a symplectic approach in which Theorem 2.1 would become a Lagrangian intersection result. Surprisingly, such an approach exists. However, it is not elementary anymore - it is based on the recent notion of symplectic quasi-states which was introduced by Entov and Polterovich in [EP1]. We refer the interested reader to [EP1, EP2, EPZ] for a detailed description of quasi-states in symplectic topology.

A symplectic quasi-state on a closed connected symplectic manifold $(M, \omega)$ is a functional

$$
\zeta: C^{0}(M, \mathbb{R}) \rightarrow \mathbb{R}
$$

satisfying the following properties:

1. $\zeta(1)=1$

2. $\zeta(F) \leq \zeta(G)$ if $F \leq G$ 
3. $\zeta(a F+b G)=a \zeta(F)+b \zeta(G)$ for all $a, b \in \mathbb{R}$ and all smooth functions $F, G$ with vanishing Poisson bracket.

It is shown in $[\mathrm{EP} 2, \mathrm{EP} 1]$ that for $(M, \omega)=\left(\mathbb{S}^{2}, \omega\right)$ with $\int_{\mathbb{S}^{2}} \omega=1$, a symplectic quasi-state $\zeta$ can be constructed as follows. For any given Morse function $F: \mathbb{S}^{2} \rightarrow \mathbb{R}$ with distinct critical values, there is a unique connected component $C_{F}$ of the level lines of $F$ such that the $\omega$-area of every connected component of its complement is at most $1 / 2$. Then set

$$
\zeta(F)=F\left(C_{F}\right)
$$

and extend it to all $F \in C^{0}\left(\mathbb{S}^{2}, \mathbb{R}\right)$ by continuity with respect to the $C^{0}$-norm. Note that $\zeta$ is invariant under any diffeomorphism of $\mathbb{S}^{2}$ which preserves the non-oriented area, in particular,

$$
\zeta(F)=\zeta(F \circ \sigma) .
$$

Now assume that $f$ is a smooth function on $\mathbb{S}^{2}$, satisfying (1) and having 0 as a regular value. Then (5), together with the linearity of $\zeta$, implies that

$$
\zeta(f)=\zeta(f \circ \sigma)=\zeta(-f)=-\zeta(f)
$$

so that $\zeta(f)=0$. But, by definition of $\zeta$, this means that $f^{-1}(0)$ contains an equator $C_{f}$, and Theorem 2.2 is proven.

\section{Concluding remarks}

If $i \neq$ id is any involution on $\mathbb{S}^{2}$ then a classical result-apparently due to Brouwer [Bro], see also [Ker] - states that $i$ is topologically conjugate to a Euclidean rotation about an angle of $\pi$, the reflection along an equatorial plane, or the antipodal map $\sigma$.

Theorem 5.1. Let $i: \mathbb{S}^{2} \rightarrow \mathbb{S}^{2}$ be any involution. Then, for every continuous mapping $V: \mathbb{S}^{2} \rightarrow \mathbb{R}^{2}$, there exists at least one point $x \in \mathbb{S}^{2}$ such that $V(x)=$ $V(i(x))$.

Proof. If $i$ has fixed points, the theorem is trivially true. If $i$ is fixed point free it is topologically conjugate to $\sigma$, and applying Theorem 1.1 yields the result.

\section{References}

[Bor] K. Borsuk: Drei Sätze über die $n$-dimensionale euklidische Sphäre, Fund. Math. 20, 177-190 (1933)

[Bro] L. E. J. Brouwer: Über die periodischen Transformationen der Kugel, Math. Ann. 80, 39-41 (1919) 
[Dod] M. M. Dodson: Chord theorems, circle maps and the Borsuk-Ulam theorem, New Zealand J. Math. 22, 23-29 (1993)

[DG] J. Dugundji, A. Granas: Fixed Point Theory, Springer 2003

[EP1] M. Entov, L. Polterovich: Calabi quasimorphism and quantum homology, Intern. Math. Res. Notices 30, 1635-1676 (2003)

[EP2] M. Entov, L. Polterovich: Quasi-states and symplectic intersections, Comment. Math. Helv. 81, 75-99 (2006)

[EPZ] M. Entov, L. Polterovich, F. Zapolsky: Quasi-morphisms and the Poisson bracket, preprint arXiv:math/0605406v2 (2007)

[Fan] K. Fan: A generalization of Tucker's combinatorial lemma with topological applications, Ann. Math. 56, 431-437 (1952)

[GP] V. Guillemin, A. Pollack: Differential Topology, Prentice Hall 1974

[Had] H. Hadwiger: Ein Satz über stetige Funktionen auf der Kugelfläche, Arch. Math. 11, 65-68 (1960)

[Hir] M. Hirsch: Differential Topology, Springer 1976

[Ker] B. v. Kerékjártó: Über die periodischen Transformationen der Kreisscheibe und der Kugelfläche, Math. Ann. 80, 36-38 (1919)

[Su] F. E. Su: Borsuk-Ulam implies Brouwer: a direct construction, Amer. Math. Monthly 104, 855-859 (1997)

[Tuc] A. W. Tucker: Some topological properties of disk and sphere, Proc. First Canadian Math. Congress 1945, 285-309, University of Toronto Press 1946 


\section{Preprints ab 2008}

2008-01

2008-02

2008-03

2008-04

2008-05

2008-06

2008-07

2008-08

2008-09

$2008-10$

2008-11
Henryk Zähle

Weak approximation of SDEs by discrete-time processes

\section{Benjamin Fine, Gerhard Rosenberger}

An Epic Drama: The Development of the Prime Number Theorem

Benjamin Fine, Miriam Hahn, Alexander Hulpke, Volkmar große Rebel, Gerhard Rosenberger, Martin Scheer

All Finite Generalized Tetrahedron Groups

Ben Schweizer

Homogenization of the Prager model in one-dimensional plasticity

Benjamin Fine, Alexei Myasnikov, Gerhard Rosenberger

Generic Subgroups of Group Amalgams

Flavius Guiaş

Generalized Becker-Döring Equations Modeling the Time Evolution of a Process of Preferential Attachment with Fitness

Karl Friedrich Siburg, Pavel A. Stoimenov

A scalar product for copulas

Karl Friedrich Siburg, Pavel A. Stoimenov

A measure of mutual complete dependence

Karl Friedrich Siburg, Pavel A. Stoimenov

Gluing copulas

Peter Becker-Kern, Wilfried Hazod

Mehler hemigroups and embedding of discrete skew convolution semigroups on simply connected nilpotent Lie groups

Karl Friedrich Siburg

Geometric proofs of the two-dimensional Borsuk-Ulam theorem 